\title{
Stress less-balloon therapy is effective for SUI
}

Data from the SOLECT trial have indicated that Vesair ${ }^{\mathrm{TM}}$ (Solace Therapeutics, USA) - a noninvasive, intravesical pressure-attenuated balloon system - is safe and effective in the treatment of women with stress urinary incontinence (SUI).

The Vesair ${ }^{\text {TM }}$ balloon directly attenuates the transient spikes in intravesical pressure that are common to all forms of SUI. This system works as a hydraulic 'shock-absorber' and uses the principle that, relative to most liquids, gas is highly compressible. Placement of the deflated balloon into the bladder is through the urethra where it is then inflated with $30 \mathrm{cc}$ of air and $0.5 \mathrm{cc}$ of AirLoc ${ }^{\mathrm{TM}}$ (Solace Therapeutics, USA) - a perfluorocarbon liquid that prevents the balloon deflating.

Wyndaele et al. report results from the prospective, multicentre, randomized, controlled SOLECT trial in Neurourology and Urodynamics. 63 women with SUI or mixed urinary incontinence with SUI as the predominant component were randomized 2:1 to receive either the balloon system (the treatment arm) or a sham procedure (the control arm). The primary outcome measure was a composite end point of $\geq 10$ increase in the 22 -item incontinence quality of life survey and a $\geq 50 \%$ decrease in pad weight (assessed under controlled, provocative conditions), after a 3-month follow-up period.

Composite end point results were available for 50 women (31 from the treatment arm and 19 from the control arm). The symptoms of women who received the Vesair $^{\mathrm{TN}}$ system improved significantly compared with those who had the sham procedure when analysed on an intent-to-treat (63.4\% versus $31.8 \%$, respectively, $P=0.02)$ and per protocol (67.7\% versus $31.6 \%$, respectively, $P=0.0195)$ basis. Significant differences were also observed in the percentage of women achieving a $\geq 50 \%$ reduction in pad weight in each group $(80.7 \%$ in the treatment arm and $45.0 \%$ in the control arm,

$P=0.0143$ ).

Notably,

$41.9 \%$ of

patients who received treatment were assessed as dry in their pad weight test (defined as $\leq 1 \mathrm{~g}$ increase in weight), compared with none who underwent the sham procedure $(P=0.0006)$. No serious adverse effects occurred during the 3-month study period but dysuria, gross haematuria and UTI were reported in the treatment group.

This noninvasive therapy could provide a safe and effective alternative treatment for women with SUI.

\section{Louise Stone}

Original article Wyndaele, J.-J. et al. A randomised, controlled clinical trial of an intravesical pressure-attenuated balloon system for the treatment of stress urinary incontinence in females. Neurourol. Urodyn. doi:10.1002/nau.22798 